\title{
Depth position errors in inclinometer surveys and false displacement results
}

\author{
PE Mikkelsen GeoMetron Inc PS, USA
}

E DiBiagio Norwegian Geotechnical Institute, Norway

\begin{abstract}
The first indication of displacement error due to incorrect positioning of a traversing inclinometer probe was, in the authors' experience, pointed out by a member of the landslide investigation team during the Clyde River Gorge project investigations at Cromwell, New Zealand in 1990. Preliminary results from one of the inclinometers indicated to the project geologists that active toe-buckling at the river level (or below) of the very high schist slope formation was occurring. After closer scrutiny, the data turned out to be false.

Since then, many such project results have been brought to the authors' attention, having experienced false displacement results due to systematic depth control changes and for the most part when nobody were cognisant of the issue. It is still very much of an unknown factor among inclinometer users, but it appears to be more of an issue now than before because of unavailability of original control cable manufacture and the introduction of thinner inclinometer cables.

Accuracy of manual, traversing inclinometer surveys is entirely dependent on probe depth position repeatability. Any deviation produces a corresponding error because the casing attitude (tilt or slope) is never perfectly linear. In fact, a preponderance of installations are helical, readings of tilt (slope) always changes with the slightest change in depth.

Two factors have to be present for depth position error (DPE) to be significant. Its magnitude is a function of curvatures in the casing (change in tilt with depth) and the vertical probe positioning error (PPE) or shift from the initial data set. In most cases, the PPE is an unknown and has to be estimated graphically or numerically. Error in the vertical positioning may occur in four ways:

1. When the casing compresses due to settlement.

2. When the control cable changes length.

3. When the reference point at the top of the casing changes.

4. When the casing is shortened or extended at the top.

How do we know that this error is present? Based on experience over the years it was discovered that there is geometric congruence between plots of displacement error and magnitude of the tilt readings with depth. The error is mathematical or systematic! Distribution of changes has to be estimated based on the geometry (curvatures) with spreadsheet back calculation analysis. The latter procedure is frustrated by presence of other errors. It is very time consuming and not always entirely perfect. GTilt and DigiPro software allow corrections for DPE by trial-and-error, but without built-in diagnostic routines to estimate PPE. Confronting $D P E$ and unknown PPE by using diagnostics and calculations is the main subject of this paper.
\end{abstract}

\section{Introduction}

Displacement error due to incorrect positioning of a traversing inclinometer probe, in the authors' experience, goes back to the landslide investigation during the Clyde River Gorge project investigations at Cromwell, New Zealand in 1990. Preliminary results from one of the inclinometers indicated to the project geologists that active toe-buckling at the river level (or below) of the very high schist slope formation was occurring. After closer scrutiny, the data turned out to be false. It was an artefact in the data caused by 
cable twist and a gradual shortening of the control cable on a storage reel. Since then, many such project results have been brought to the authors' attention, having experienced false displacement results due to systematic depth control changes and for the most part when nobody was cognisant of the issue. It is still very much of an unknown factor among inclinometer users, but it appears to be more of an issue now than before because of unavailability of original control cable manufacture and the introduction of thinner inclinometer cables. The old cable design for oceanography research from the 1950s is no longer being produced. Reinforced electrical cable designs similar to the old design do not seem to come up to the old standard. Thinner cables have also been introduced, but thinner cables have less length stability than most have anticipated. It can be a big source of error for installations over $30 \mathrm{~m}$ depth.

Accuracy of manual, traversing inclinometer surveys is dependent on probe depth position repeatability. Any deviation produces a corresponding error because the casing attitude (tilt) is never perfectly linear. In fact, a preponderance of installations are helical, readings of slope (tilt) always changes with the slightest change in depth.

Two factors have to be present for depth position error (DPE) to be significant. Its magnitude is a function of curvatures in the casing (change in tilt or slope with depth) and the vertical probe positioning error (PPE) or shift from the initial data set.

How do we know that this error is present? Based on experience over the years it was discovered that there is geometric congruence between plots of displacement error and magnitude of the tilt readings with depth (Mikkelsen 2003). The error is mathematical (systematic)! Graphically, plots of displacements generated by DPE will be geometrically congruent with plots of magnitude of the tilt readings with depth. It is the best diagnostic tool available in this regard. Rescaling the two plots of displacement and tilt readings vs. depth to be congruent, directly or mirror image, goes a long way to estimate the PPE. Once the PPE is estimated corrections to the DPE afflicted data set can be made either by spreadsheet or DigiPro or GTilt software.

Error in the vertical positioning may occur in four ways:

1. When the casing compresses due to settlement.

2. When the control cable changes length.

3. When the reference point at the top of the casing changes.

4. When the casing is shortened or extended at the top.

Examples of the first two types will be present in this paper. The last two types are simplified versions of the first two.

\section{Terminology and error mathematics}

A number of terms are used to describe inclinometer data and calculated results. This paper uses mainly terms used in DigiPro2 software, but equivalent historic terms are also described to help comprehension. Tilt is the term for fundamental inclinometer output and is expressed in a number of ways. Accelerometer output from the inclinometer probe is calibrated in terms of sine of the slope angle from vertical (gravity) times an instrument constant. Historically, the instrument constant was an observer convenience factor so that $1 / 10$ th of a millivolt output resolution and the probe gauge length would combine to an integer expressing deviation from vertical. When displayed raw readings are converted to engineering units for one reading interval it was called incremental deviations (from vertical), the same as slope and tilt. It was expressed mostly as reading units in the past, but current computer based readout/recorders have options for outputs in engineering units like $\mathrm{mm}, \mathrm{m}$, inches and feet. A plot of the tilt with depth is a fundamental diagnostic tool for detecting DPE, a tool not in the normal order of inclinometer data reduction and presentation. It is a plot of the readings with depth and is a unique characteristic of each inclinometer casing installation. It will simply be referred to as a tilt plot. 
A cumulative displacement plot is the most common result of inclinometer data reduction. This plot is often referred to as simply a displacement plot, deflection or movement plot. More recent terminology is profile change. The profile of each inclinometer installation is unique and forms the baseline for subsequent readings of the change in this profile. Any type of change in tilt readings at each interval will look like displacement. When the vertical probe position is changed from its initial survey the PPE produces DPE in the displacement plot. It is superimposed on actual displacements and on other systematic errors.

The DPE is a result of a simple mathematical relationship between PPE and change in tilt over the interval considered. The change in tilt or slope is simply curvature by mathematical definition. It can be expressed for one interval as follows:

$$
D P E=(P P E / L) \times C
$$

where:

$$
\begin{array}{lll}
\mathrm{PPE} & = & \text { the probe position error. } \\
\mathrm{L} & = & \text { the probe length }=\text { reading interval. } \\
\mathrm{C} & = & \text { the curvature }=\mathrm{T} 2-\mathrm{T} 1 . \\
\mathrm{T} 1 & = & \text { tilt at the original level of reading. } \\
\mathrm{T} 2 & = & \text { tilt at the interval below or above depending on the polarity of the PPE. }
\end{array}
$$

As an example, assume PPE $=100 \mathrm{~mm}, \mathrm{~L}=500 \mathrm{~mm}$ and $\mathrm{C}=(6.12-3.02) \mathrm{mm}$ the error according to Equation 1 in one interval would be:

$$
\operatorname{DPE}(\mathrm{mm})=(100 / 500) \times(6.12-3.02)=0.20 \times(3.10)=0.62 \mathrm{~mm}
$$

From the above example, it is then plain to understand that a PPE of $100 \mathrm{~mm}$ persisting over a constant curvature for $5 \mathrm{~m}$ of depth would accumulate to a total DPE of $6.2 \mathrm{~mm}$. For deeper borings over $30 \mathrm{~m} \mathrm{drift}$ off vertical is quite common and often have constant curvature with depth. Boring drift has little consequence for exploration drilling, but can have serious consequences for inclinometer results, particularly those programs spanning several years with changes in equipment, personnel and site conditions.

As a side note, and perhaps of secondary importance in the authors' opinion, is that the mathematics above assumes a linear progression of curvature from one reading interval to the next (circular). That is not exactly correct and introduces a small, residual 'ripple' in the corrected results. But so far, it is the best assumption that can be made with a reading resolution equal to the length of the probe being $0.5 \mathrm{~m}, 1 \mathrm{~m}$ or $2 \mathrm{ft}$.

\section{The PPE problem}

In the authors' experience a plan to measure vertical ground effects such as settlement with depth in conjunction with the inclinometer program is rather rare. Similarly, routine calibrations of the control cable to ascertain its stability is practically non-existent. This leaves us with most situations confronting DPE with the PPE unknown and having to use graphic diagnostics and spreadsheet calculations to estimate it. That is the main theme of this paper.

However, if the PPE is measured, correcting for DPE is rather straight forward. With a bit of foresight and planning, steel bands or magnetic strips can be installed on the inclinometer casing exterior at broad intervals of 3 to $6 \mathrm{~m}$ or more. The vertical depth to these 'targets' can be measured with instruments such as a Sodex or a MagEx. These instruments are of course only as accurate as the stability of their measuring cables. All measuring cables should be periodically calibrated, since contemporary cable designs cannot be counted on to be stable and accurate long-term. 


\section{Effect of settlement, example 1}

Inclinometer casings installed through fill over compressible ground is often subjected to significant vertical compression due to down-drag from settlement. Typically, the casing is installed in borings through the fill and the compressible soil extending into underlying relatively stable ground. As the soil consolidates with time, both the soil and the overlying fill drags the ABS plastic inclinometer casing down to varying degrees and shortens it; the distance between the top and bottom ends diminishes. Generally, shortening of up to $1 \%(300 \mathrm{~mm}$ in $30 \mathrm{~m})$ does not adversely affect the mechanical integrity of the casing (note that if shortening of over $1 \%$ is anticipated, telescoping couplings must be installed in the compressible zones). However, it can introduce data errors when readings are taken at normally fixed, $0.5 \mathrm{~m}$ or $2 \mathrm{ft}$ intervals. For fixed reading intervals the probe will be set at a gradually lower depth positions in the casing as settlement or compression occurs. For the typical fill scenario in Figure 1, the errors in the depth settings would start to accumulate from the top down, readings starting just below the compressible soil having the highest potential for errors. In other words, the worst errors are likely to be found where stable conditions are expected.

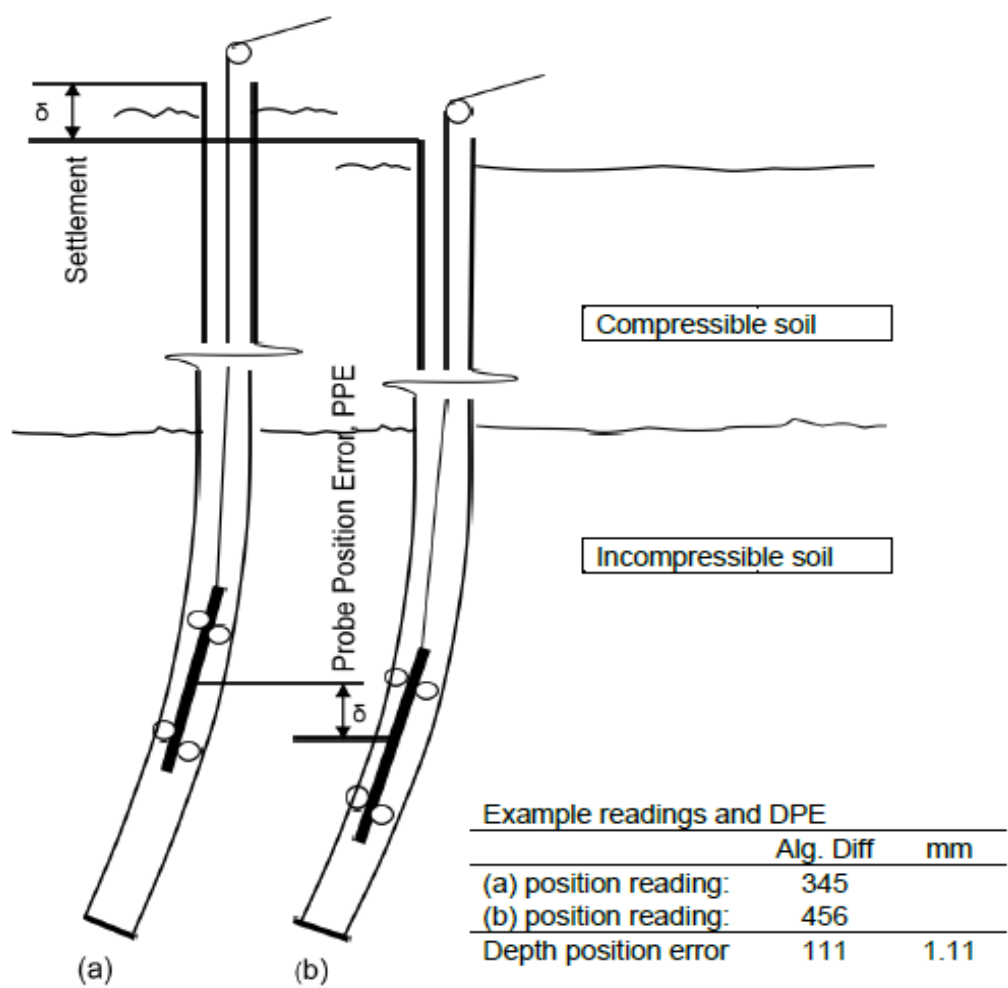

Figure 1 Illustration of depth position error (DPE) in casing with curvature caused by settlement; (a) initial probe position with tilt reading of $3.45 \mathrm{~mm}$; and (b) subsequent probe position after settlement with tilt reading of $4.56 \mathrm{~mm}$. DPE = $1.11 \mathrm{~mm}$

The illustration in Figure 1 shows the problem situation. The bottom reading will see the full effect of settlement $\delta$. The probe position error at that depth will be equal to the settlement of that depth. 
The term 'potential for errors' is used because a systematic error of this nature is a product of two factors where both have to be present to produce it. If the casing is perfectly linear in shape with depth (readings are constant) there will be no error. A casing with curvatures (readings change with depth) will produce errors proportional to the amount of curvature and the amount of depth position error at each interval.

FILL2 SI-7 A

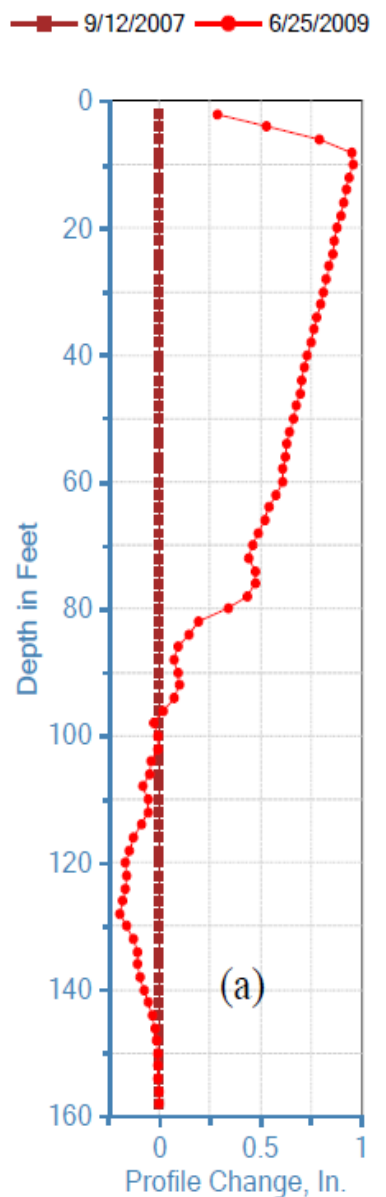

FILL2 SI-7 A

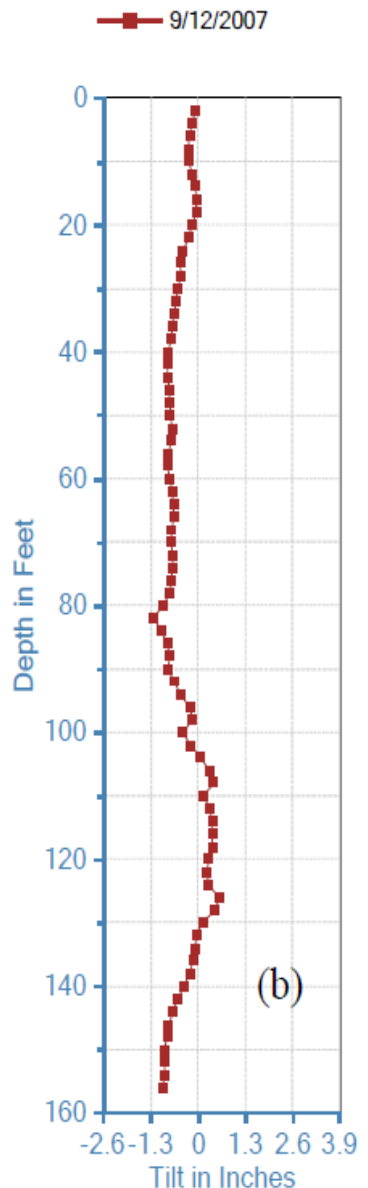

FILL2 SI-7 A
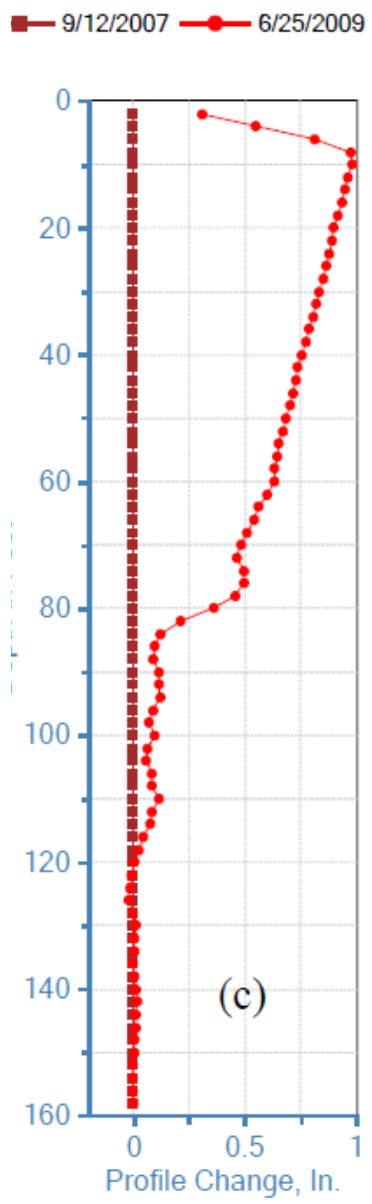

Figure 2 Example 1: depth position error (DPE) caused by settlement; (a) uncorrected plot; (b) tilt reading plot; and (c) plot corrected for DPE

Example 1 in Figure 2 first illustrates how to compare the uncorrected change in the profile suspected of DPE (a) with the plot of tilt for the casing (b). The soil stratification is similar to the schematic in Figure 1. This is actual project data that was measured in English units, not easily converted to metric. It is clear that the changes from ground surface through fill to $100 \mathrm{ft}(30 \mathrm{~m})$ depth of $1^{\prime \prime}(25 \mathrm{~mm})$ are credible. The tilt plot (b) is vertical in this interval and there is no congruence. But the $1 / 4$ " $(6 \mathrm{~mm})$ change below $120 \mathrm{ft}(40 \mathrm{~m})$ depth is suspect and there is mirror congruence with the tilt plot. The scales have been optimised to make 
the horizontal graphics as close to the same width as possible below $120 \mathrm{ft}(40 \mathrm{~m})$ depth. Thus the ratio of (a) scale for profile change and (b) scale for tilt is about 0.2 . The PPE is then $0.2 \times 2 \mathrm{ft}=0.4 \mathrm{ft}$ of settlement. The mirror image congruence means the probe is at intervals below the original reading intervals. By uniformly applying the value of $0.4 \mathrm{ft}$, below $80 \mathrm{ft}$ depth, to the settlement routine for DPE caused by settlement in DigiPro2 software the corrected results in plot (c) emerges. In this case it does not fundamentally change the main results, but it eliminates the uncertainty about no displacements below $120 \mathrm{ft}$ depth.

\section{$5 \quad$ Effect of cable stretch, example 2}
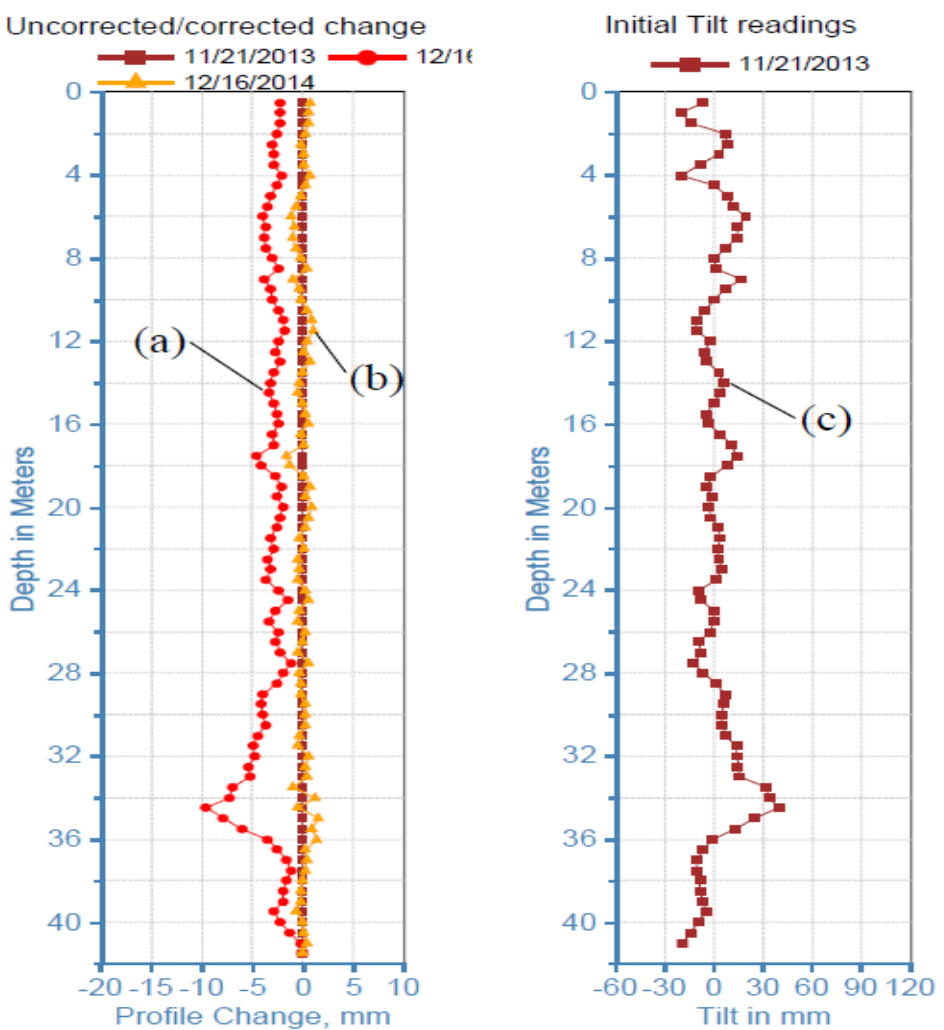

Figure 3 Example 2: illustration of uncorrected and corrected depth position error (DPE); (a) uncorrected displacement plot with DPE; (b) corrected displacement plot; and (c) plot of the tilt readings

Many examples of cable length change have been encountered over the last 15-20 years by the authors. Example 2 is from another project from the authors' recent files. The PPE was estimated by first scaling the change in profile (a) equivalent to the mirror image of the tilt plot (c). The ratio of the two scales was $20 / 120=0.167$, a PPE of $500 \times 0.0167=83 \mathrm{~mm}$. This PPE value was initially tried uniformly below $20 \mathrm{~m}$ depth. It eliminated the DPE below $34.5 \mathrm{~m}$ depth, but over-corrected it above. The PPE was adjusted to lower values by trial-and-error in DigiPro2 so that the DPE was minimised to the results shown as curve (b) in Figure 3. The original and changed cable lengths used are shown in Table 1. 
Table 1 Correction values utilised for DPE in Example 2, Figure 3

\begin{tabular}{cc}
\hline Selected original depths $(\mathrm{m})$ & $\begin{array}{c}\text { Depths adjusted for PPE }(\mathrm{m}) \\
\text { (cable stretch) }\end{array}$ \\
\hline 0.5 & 0.5 \\
20 & 20 \\
28 & $28-0.080=27.92$ \\
34.5 & $34.5-0.083=34.417$ \\
40.0 & $40-0.083=39.917$ \\
\hline
\end{tabular}

\section{Conclusion}

There is no automatic correction for errors caused by changes in the depth position of the inclinometer probe. With foresight and planning, the changes can be measured and corrections made using those results. This is rarely done.

The DPE normally appears unexpectedly. Therefore, the DPE has to be corrected by trial-and-error. Displacement results, particularly below $30 \mathrm{~m}$ depth, should be scrutinised for DPE. The diagnostic plot of tilt with depth should be made and compared to changes or displacement plots for each axis. Congruence between the two plots, even partially, should be taken as a sign of DPE. It is entire unreasonable that actual displacements would be proportional to the magnitude of the readings.

The suspected DPE can be corrected once the PPE has been estimated from the results. This is done by graphical comparisons, simple calculations and some trial-and-error for 'best fit' to minimise the DPE using DigiPro (http://www.slopeindicator.com) or GTilt (http://www.mitresoftware.com) software. Two examples were discussed in this paper.

\section{Acknowledgement}

My career and insight into systematic error aspects of inclinometer data would not exist without my mentor, the late Stanley D Wilson, inventor of the slope indicator and the modern accelerometer based inclinometer systems. I am also indebted to the technical personnel I worked with in Cromwell, New Zealand from 1989 to 1992 . Their critical evaluation of data led to early detection and correction of a unique form of cable shortening. Great contributions to software development with GTilt came from John Graham with Mitre in Edmonton, Canada during the work at Cromwell. Shortly after, personnel at Slope Indicator Company (DGSI) developed DMM/DigiPro and later yet, DigiPro2 including corrections for DPE and other systematic errors. I am grateful to Tom Spaulding, Dale Shoup and Jim McCool at Slope Indicator for their contributions. Special thanks also go to my co-author, Dr Elmo DiBiagio, formerly with the Norwegian Geotechnical Institute for his valuable insight and critique of former narratives on DPE while collaborating on a large mine waste project.

\section{References}

Mikkelsen, PE 2003, 'Advances in inclinometer data analysis', in F Myrvoll (ed.), Proceedings of the 6th International Symposium on Field Measurements in Geomechanics, A. A. Balkema, Rotterdam, pp. 555-567. 
